\title{
Duplexer using microwave photonic band gap structure
}

\author{
Sang Soon Oh, Chul-Sik Kee, ${ }^{\text {a) }}$ Jae-Eun Kim, and Hae Yong Park ${ }^{\text {b) }}$ \\ Department of Physics, Korea Advanced Institute of Science and Technology, Taejon 305-701, Korea \\ Tae II Kim, Ikmo Park, and H. Lim \\ Department of Molecular Science and Technology and Division of Electrical Engineering, Ajou University, \\ Suwon 442-749, Korea
}

(Received 30 August 1999; accepted for publication 18 February 2000)

\begin{abstract}
We propose a frequency selective duplexer using microwave photonic band gap (PBG) structures. It uses two different PBGs to control the propagation of electromagnetic waves in the microwave region. In this structure, an additional narrow reflection band appears in the transmission spectrum when the PBG structure is not properly located relative to the $T$ junction. By considering multiple reflections, it is proved that this additional reflection band in each PBG structure results from the interference between the input wave and the reflected wave from the other PBG structure. An effective way to prevent this interference effect is also discussed. (C) 2000 American Institute of Physics. [S0003-6951(00)04316-3]
\end{abstract}

In recent years, photonic band gap (PBG) structures, which are artificial periodic patterns of condensed matter, have received great attention. ${ }^{1}$ Plenty of new physics can be sought from the analogy between the electromagnetic (EM) waves traveling in PBG structures and the electron waves propagating in crystals. ${ }^{1}$ Moreover, since the properties of PBG structures are scalable, they have a wide range of potential applications from light wave devices such as lightemitting diodes, laser diodes, and omnidirectional reflectors $^{2-4}$ to microwave devices such as antennas and power amplifiers. 5,6

It is widely known that a strong periodic variation in the dielectric permittivity $\epsilon$ or refractive index $\sqrt{\mu \epsilon}$ gives rise to PBGs. ${ }^{1,7} \mathrm{We}$, however, have shown recently that the basic parameter is not the periodic variation of the refractive index but that of wave impedance $\sqrt{\mu / \epsilon}$, i.e., the characteristic impedance of the structure. ${ }^{8}$ This suggests that the structural matching between different PBG structures would affect the transmission characteristics of the PBG structure. To our knowledge, however, the problem related to matching of microwave PBG structures has not been studied. In this letter, we report the influence of structural matching, i.e., the distance between the PBG structure and the junction point, on the PBG transmission characteristics. Frequency selective switching behavior of PBG is also discussed.

The PBG structures employed are one-dimensional (1D) lattices with square cells and two-dimensional (2D) square lattices with square cells. The PBG structures are formed by etching the ground plane of a $50 \Omega$ microstrip line (Fig. 1), since they are also known to function as PBG structures. ${ }^{8,9}$ The substrate used is RT/Duroid 6010 which has a dielectric constant of 10.2 and thickness of $0.635 \mathrm{~mm}$. The period $a$ is chosen so that the PBG operates in the $5-15 \mathrm{GHz}$ range. The transmission characteristics of the PBG structures are simulated using ENSEMBLE which is a full wave EM simulator

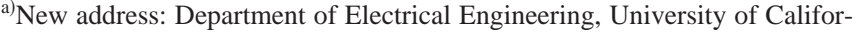
nia, Los Angeles, Los Angeles, California 90095.

${ }^{b)}$ Electronic mail: hypark@ sorak.kaist.ac.kr
}

based on the method of moments and measured experimentally using an HP8753 network analyzer.

The T-junction type PBG structure shown in Fig. 1 consists of PBG1 and PBG2 whose periods, and therefore stop bands, are quite different from each other. When the input signal coming from port 3 has frequencies that belong to the stop bands of PBG1 and PBG2 the signal corresponding to PBG1 would go to port 2 and that of the PBG2 to port 1, since each PBG structure perfectly reflects the signals corresponding to its stop band. Thus, this T-junction type of PBG structure functions as a frequency selective duplexer which divides two signals of different frequency into different paths. Quite surprisingly, however, another sharp stop band appears in the transmission spectrum of each PBG structure as shown in Fig. 2 for the case of $d_{1}=8.2 \mathrm{~mm}$ and $d_{2}$ $=11.0 \mathrm{~mm}$. Note that this additional sharp stop band appearing in the transmission spectrum of each PBG structure falls into the frequency range of the principal stop band of the other PBG structure. Moreover, we observed that these sharp

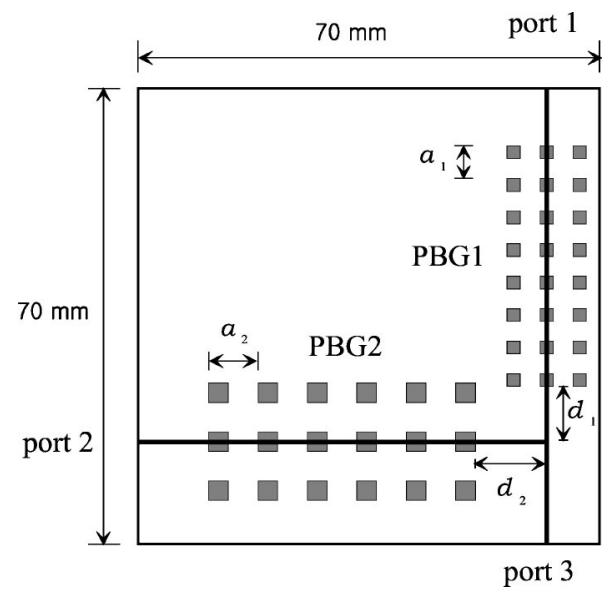

FIG. 1. T junction with a square array patterned in the ground plane. $d_{1}$ and $d_{2}$ are the distances between the junction and the corresponding PBG structures. The periods are taken to be $a_{1}=5 \mathrm{~mm}$ and $a_{2}=7.5 \mathrm{~mm}$. The cell sizes are $2 \times 2 \mathrm{~mm}^{2}$ and $3 \times 3 \mathrm{~mm}^{2}$ for the PBG1 and PBG2 structures, respectively. 


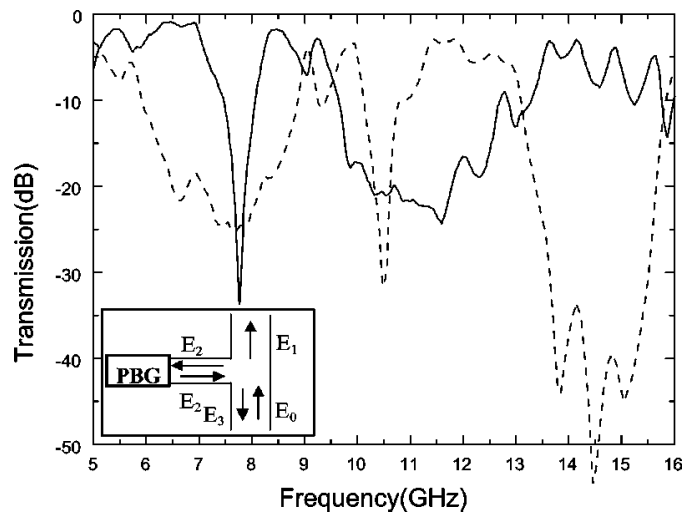

FIG. 2. Measured transmission characteristics for $d_{1}=8.2 \mathrm{~mm}$ and $d_{2}$ $=11 \mathrm{~mm}$. Note that an additional stop band appears in the transmission spectrum of each PBG structure in the frequency range of the principal stop band of the other PBG structure. The solid line is for $S_{13}$ and the segmented one line is for $S_{23}$. The inset shows the wave components.

bands disappear when either the PBG1 or PBG2 structure is removed. Therefore, it is evident that the sharp stop band in the transmission spectrum of each PBG structure is due to the wave reflected from the other PBG structure. On the other hand, a broad stop band appearing above $13 \mathrm{GHz}$ in the $\mathrm{S}_{23}$ spectrum remains even though the PBG1 structure is removed, and that is evidently the second harmonic of the principal stop band of the PBG2.

To understand the formation of the additional stop band, we analyzed the wave reflections at the junction and the PBG structure. Because a wave with the amplitude $E_{0}$ is incident from the port, it passes a $50 \Omega$ microstrip line to the $\mathrm{T}$ junction and then is divided into two parallel $50 \Omega$ microstrip lines. The reflection coefficient $r=-1 / 3$ and the transmission coefficient $t=2 / 3$ at the junction, and the wave $E_{0}$ is divided into three waves of $E_{1}=2 E_{0} / 3, E_{2}=2 E_{0} / 3$ and $E_{3}$ $=-E_{0} / 3$ as shown in the inset of Fig. $2 .{ }^{10}$ Each PBG structure behaves as a perfect reflector or transmitter depending on the frequency that falls in or out of its stop band, respectively. Thus the wave component reflected from PBG2 undergoes a partial reflection at the $\mathrm{T}$ junction with a reflection coefficient $r=-1 / 3$, and then it is perfectly reflected again by PBG2, and so on. The amplitude of the waves going to port 1 and port 3, after multiple reflections at PBG2, is given by

$$
\begin{aligned}
E_{r} & =E_{0}\left[\frac{2}{3} e^{i 2 \phi}+\frac{2}{3}\left(-\frac{1}{3}\right) e^{i 4 \phi}+\frac{2}{3}\left(-\frac{1}{3}\right)^{2} e^{i 6 \phi}+\ldots\right] \\
& =\frac{4}{9} E_{0} \frac{e^{i 2 \phi}}{1+\frac{1}{3} e^{i 2 \phi}},
\end{aligned}
$$

where $\phi$ is the phase delay due to the optical path length of $d_{2}$. This equation is very similar to that of a reflected wave in an asymmetric Fabry-Pérot interferometer made of a perfect mirror and low reflection mirror, ${ }^{11}$ where the amplitude of reflected wave is given as $E_{r}=E_{0} t^{2} e^{i 2 \phi} /\left(1-r e^{i 2 \phi}\right)$. This similarity is expected, since a perfect reflection at one end and low reflection at the other end occur in both systems.

The phase $\phi$ in Eq. (1) is simply given as $\phi$ $=2 \pi n_{\text {eff }} d / \lambda$. Here, $n_{\text {eff }}$ is the effective refractive index of the microstrip line obtained from the effective dielectric constant Downloaded 27 Oct 2006 to 143.248.16.71. Redistribution subjec

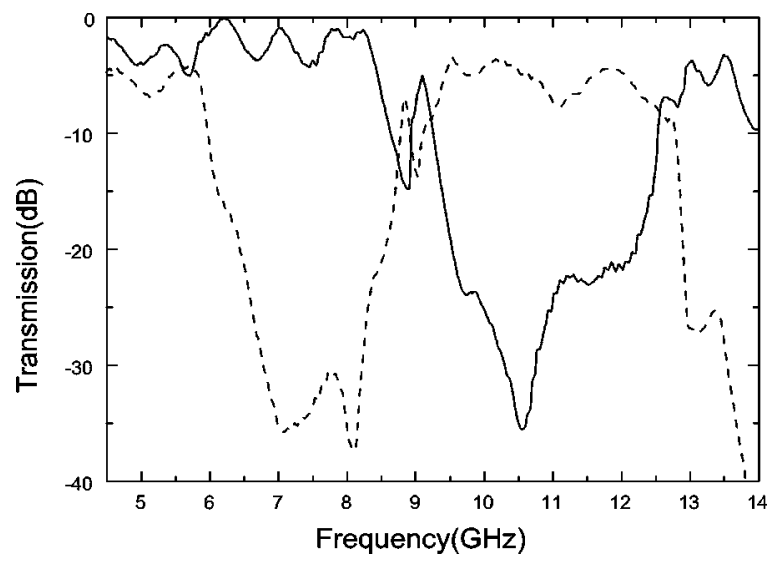

FIG. 3. Measured transmission characteristics for $d_{1}=d_{2}=1.2 \mathrm{~mm}$. The solid line is for $\mathrm{S}_{13}$ and the segmented line is for $\mathrm{S}_{23}$.

$\epsilon_{\text {eff }}$ of the microstrip line. When this phase delay is $\phi=(m$ $-1 / 2) \pi$ where $m$ is an integer, the frequency $f_{m}$ is given by

$$
f_{m}=\left(m-\frac{1}{2}\right) \times \frac{c}{2 n_{\mathrm{eff}} d} .
$$

and $E_{r}$ becomes $-2 E_{0} / 3$. Thus we obtain $E_{1}+E_{r}=0$ in the direction of port 1 and $E_{3}+E_{r}=-E_{0}$ in the direction of port 3. This means that the input wave from port 3 is totally reflected and no transmission is allowed in the direction of the ports 1 and 2 for the wave of frequency $f_{m}$. When $\phi$ $=m \pi, E_{r}$ becomes $E_{0} / 3$. Now the wave in the direction of port 1 is $E_{0}$, and that of port 3 is zero, leading to perfect transmission to the port 1 . When $\phi$ is not exactly $\pi$, a portion of the wave will be transmitted to port 1 because $E_{r}$ cannot cancel $E_{1}$ exactly. Therefore, a stop band of considerable size exists only around the frequencies satisfying Eq. (2).

The mode frequencies in Eq. (2) can also be obtained by simple modal analysis. ${ }^{12}$ These frequencies are the resonant modes of a resonator that has a closed end at the PBG structure and an open end at the junction. The electric field has its maximum value at the open end and interferes destructively with the wave going to port 1 because of the total phase delay of $2 \phi=\pi$. When the width of microstrip line, $w$ $=0.6 \mathrm{~mm}$, is smaller than the thickness of substrate $h$ $=0.635 \mathrm{~mm}$, i.e., $w / h<1$, the effective dielectric constant $\epsilon_{\text {eff }}$ is given by ${ }^{10}$

$$
\epsilon_{\mathrm{eff}}=\frac{\epsilon_{1}+\epsilon_{2}}{2}+\frac{\epsilon_{1}-\epsilon_{2}}{2}\left(1+\frac{12 h}{w}\right)^{-1 / 2},
$$

where $\epsilon_{1}$ and $\epsilon_{2}$ are the dielectric constants of the substrate and air, respectively. Using the values of $\epsilon_{1}=10.2, \epsilon_{2}$ $=1.0, d_{2}=11.0 \mathrm{~mm}$, and $m=2$, the additional stop band in $\mathrm{S}_{13}$ is calculated to be $f_{2}=7.8 \mathrm{GHz}$. With the values of $d_{1}$ $=8.2 \mathrm{~mm}$ and $m=2$, the additional stop band in $\mathrm{S}_{23}$ spectrum at $f_{2}=10.5 \mathrm{GHz}$, is predicted. The excellent agreement between these values of $f_{2}$ and the measured frequencies of the additional stop bands in Fig. 2 justifies our discussions in the preceding paragraphs on the origin of additional stop bands. In Fig. 2 we cannot see the first resonant mode $\left(f_{1}\right)$ below the frequency $f_{2}$. This is obvious since the waves of the first mode exist outside the stop bands so that they do not experience reflection from the PBGs.

to AIP license or copyright, see http://apl.aip.org/apl/copyright.jsp 

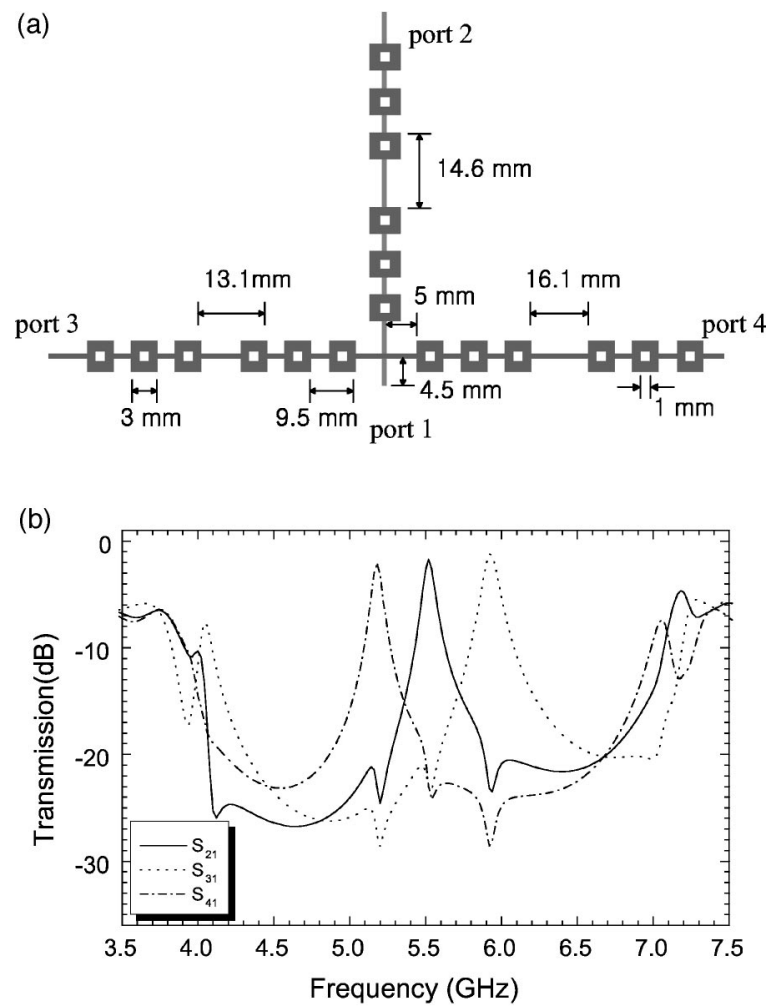

FIG. 4. Multiplexer based on the PBG structures printed on the microstrip lines (a) and calculated transmission characteristics (b). Note the narrow pass bands are created by the defect modes.

The spurious stop band due to the resonant modes can be eliminated by appropriately choosing the distances $d_{1}$ and $d_{2}$. Since the mode spacing is inversely proportional to $d_{1}$ or $d_{2}$ value [Eq. (2)], more resonant modes appear in the region concerned, if $d$ 's become longer. Thus these modes can be pushed outside the frequency region of interest only by making $d$ 's shorter. Figure 3 shows an example of the resonant mode exclusion by making $d_{1}=d_{2}=1.2 \mathrm{~mm}$. In this case, one of the overlapping cells is eliminated so that PBG1 and PBG2 do not overlap. This does not affect the transmission characteristics of PBGs, since the fields are highly localized near the microstrip line.

The duplexer proposed above uses two different PBGs directly to control propagation of the incident wave, and each PBG has a broad stop band of about $3 \mathrm{GHz}$. A duplexer or a multiplexer with a narrow pass band can also be made of defect modes of PBGs. An example is shown in Fig. 4(a). where all the PBG structures have the identical stop band and each structure has a different defect mode. Here, different defect modes are introduced by changing the defect lengths differently in the PBG structures of the same period. The simulated transmission characteristics show the PBG of 4-7 GHz and the defect modes of 5.2, 5.5 and 5.9 GHz for each structure. In this case, the problem related to the resonant mode is no longer serious, because any wave component reflected from the port is automatically rejected by the other ports of the same stop band.

In summary, we proposed a type of frequency selective duplexers and investigated their transmission characteristics. The duplexer uses two different PBG structures with broad stop bands. When the PBG structures are not properly located with respect to the $\mathrm{T}$ junction, multiple reflections between the $\mathrm{T}$ junction and PBG structures results in an unwanted additional stop band. We also discussed a multiplexers with one broad stop band and several narrow defect modes.

The authors appreciate the fruitful discussions with W. J. Byun and the technical support from Professor S. Y. Shin and H. C. Song.

${ }^{1}$ J. D. Joannopoulos, R. D. Meade, and J. N. Winn, Photonic Crystals (Princeton University Press, Princeton, 1995).

${ }^{2}$ E. Yablonovitch, Phys. Rev. Lett. 58, 2059 (1987).

${ }^{3}$ S. John, Phys. Rev. Lett. 58, 2486 (1987).

${ }^{4}$ Y. Fink, J. N. Winn, S. Fan, C. Chen, J. Michel, J. D. Joannopoulos, and E. L. Thomas, Science 282, 1679 (1998).

${ }^{5}$ T. J. Ellis and G. M. Rebeiz, IEEE MTT-S Int. Microwave Symp. Dig., 1157 (1996).

${ }^{6}$ V. Radisic, Y. Qian, and T. Itoh, IEEE Microwave Guid. Wave Lett. 8, 13 (1998).

${ }^{7}$ J. D. Joannopoulos, P. R. Villeneuve, and S. Fan, Nature (London) 386, 143 (1997).

${ }^{8}$ C.-S. Kee, J.-E. Kim, H. Y. Park, S. J. Kim, H. C. Song, Y. S. Kwon, N. H. Myung, S. Y. Shin, and H. Lim, Phys. Rev. E 59, 4695 (1999).

${ }^{9}$ V. Radisic, Y. Qian, R. Coccioli, and T. Itoh, IEEE Microwave Guid. Wave Lett. 8, 69 (1998).

${ }^{10}$ D. M. Pozar, Microwave Engineering, 2nd ed. (Wiley, New York, 1998), Chap. 3.

${ }^{11}$ P. Yeh, Optical Waves in Layered Media (Wiley, New York, 1988), Chap. 7.

${ }^{12}$ J. Danglot, J. Carbonell, M. Fernandez, O. Vanbesien, and D. Lippens, Appl. Phys. Lett. 73, 2712 (1998). 